m/s

médecine/sciences $1999 ; 15: 591-3$

\title{
THÉRAPIE GÉNIQUE : DU RÊVE À LA (DURE) RÉALITÉ SCIENTIFIQUE
}

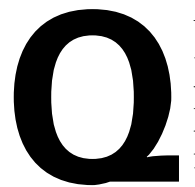

uinze ans... l'idée de transférer dans des cellules un gène codant pour une protéine potentiellement thérapeutique, et la définition des premiers vecteurs susceptibles d'opérer ce transfert, ne datent pas, ne datent plus, d'hier. D'ailleurs, le premier transfert d'un gène (marqueur) chez l'homme a eu lieu il y a dix ans [1], et la première tentative de correction d'un déficit génétique 9 ans [2]. Entourée d'un énorme intérêt médiatique (qui frise parfois le déchaînement), la thérapie génique a acquis une aura populaire comme bien peu de domaines scientifiques avant elle. Pourtant, et les synthèses présentées dans ce numéro de médecine/sciences en témoignent chacune à leur façon, l'application thérapeutique du transfert de gène est loin d'être une réalité. Alors que des moyens humains et matériels considérables ont été dévolus à la mise en œuvre de programmes de recherche visant à créer les conditions de la thérapie par transfert de gène, les bénéfices cliniques se font attendre voire, pour certains scientifiques, commencent à ne plus vraiment être attendus.

Il faut sûrement se garder d'une analyse superficielle qui ne prendrait en compte qu'un rapport comptable, investissements/bénéfices, d'une aventure qui est d'abord scientifique c'est-à-dire, par essence, soumise à un cheminement non prédictible. L'heure du bilan n'est d'ailleurs sans doute pas encore arrivée, et ce numéro de médecine/sciences n'a pas la prétention de le tirer. Il faudra, le moment venu, bien plus que ce que notre revue peut proposer : une ana- lyse scientifique exhaustive et un débat de la communauté scientifique bien plus large, encore, que celui qui avait conduit en 1995 aux États-Unis $\mathrm{au}$ fameux « rapport Varmus » [3], commandé à l'époque à un vaste panel d'experts par le Directeur des NIH. On peut noter, à ce propos, que les conclusions de ce rapport, qui appelaient en même temps à un maintien de l'effort de recherche fondamentale et à un moratoire sur les essais cliniques, sont restées lettre morte, signe sans doute du caractère prématuré d'un bilan dont les termes, en fin de compte, ne pouvaient de ce fait être acceptés. médecine/sciences propose donc ici non pas un bilan mais déjà un état des lieux tel que peuvent le réaliser des chercheurs qui, chacun dans leur discipline propre, ont poussé les recherches de transfert de gène à visée thérapeutique aux confins de l'expérience préclinique, et pour certains jusqu'aux essais cliniques pilotes.

De cet état des lieux, on peut tirer malgré tout quelques indications générales sur ce champ de recherche. Tout d'abord, ce champ qui n'existait pas il y a 15 ans, qui émergeait à peine il y a encore dix ans, a attiré à lui des centaines de laboratoires de par le monde, des milliers de chercheurs, et a donc donné naissance à des résultats scientifiques tout à fait considérables en nombre comme en qualité. Dans ce cadre, on doit tout d'abord faire une place à part à la création d'un domaine de recherche nouveau, celui de la «vectorologie ». Les acteurs de cette discipline originale visent à identifier de nouveaux vecteurs de transfert de gène, qu'ils soient viraux ou non viraux, à les 
développer dans l'optique d'applications thérapeutiques précises aussi bien que multiples et, éventuellement, à les produire dans des quantités et des conditions adaptées à l'usage clinique. On doit souligner, car il s'est agi d'une transformation majeure du mode de fonctionnement de notre communauté scientifique, le rôle complémentaire que jouent dans ce domaine des sociétés de biotechnologie spécialisées, comme la société Transgène à Strasbourg, et des centres académiques, tels ceux mis en place en France par l'Association Française contre les Myopathies à Évry, Nantes et Marseille. L'identification du champ de recherche en thérapie génique s'est également concrétisée, rapidement, par l'organisation de sociétés savantes importantes, en Europe comme en Amérique du Nord, et par le lancement de plusieurs journaux.

Même si l'on doit un jour écarter finalement le rêve d'une thérapie génique, et donc renoncer au moins partiellement à l'investissement direct qui a été réalisé dans ce champ de recherche, la mobilisation des énergies et des compétences en vue de l'élaboration de nouvelles approches pour le traitement de maladies pas ou mal soignées a marqué profondément la science biologique de cette dernière décennie. D'abord, enfant naturel de l'étude des génomes, on peut affirmer que la recherche sur la thérapie génique a grandement contribué à faire pénétrer les techniques et les approches de la biologie et de la génétique moléculaire dans les laboratoires de recherche biomédicaux, lorsqu'elles n'y étaient pas encore présentes. On peut espérer, même s'il est encore difficile d'en évaluer pleinement l'ampleur, qu'elle a (ou aura) un rôle semblable dans nombre de services hospitaliers. Cela est surtout vrai aux États-Unis, cependant, où plusieurs centaines de services cliniques ont d'ores et déjà participé, directement ou indirectement, à des essais cliniques de transfert de gène, en particulier dans le domaine du cancer. Ensuite, les retombées scientifiques des travaux visant à la mise au point de techniques de thérapie génique sont certainement majeures dans les domaines de la virologie et de la biologie moléculaire. Dans le cadre de l'identification, et de l'optimisation de nouveaux vecteurs, des virus ont ainsi été analysés, déchiffrés, suivis dans leur cycle de vie, enrichissant les concepts de l'ensemble de la discipline. En parallèle, de nombreuses études ont été sinon entreprises du moins développées sur l'utilisation de divers promoteurs naturels, et sur la mise au point de promoteurs ciblés ou contrôlés. Sans lien direct avec une perspective thérapeutique, le champ d'application des outils créés par les recherches sur la thérapie génique s'étend aujourd'hui largement dans les laboratoires de biologie cellulaire, pour exprimer sélectivement ou bloquer l'expression d'un gène, dans ceux qui étudient la biologie du développement, dans lesquels les vecteurs rétroviraux ont fait leur apparition pour suivre des lignages cellulaires il y a déjà bien longtemps [4], ou même dans les laboratoires de physiologie, pour réaliser l'analyse morpho-physiologique [5] ou fonctionnelle des cellules en culture et, de plus en plus, chez l'animal in vivo [6]. Enfin, et ce n'est certainement pas la moindre des retombées scientifiques des recherches sur la thérapie génique, la perspective (même lointaine) d'un traitement s'est ouverte pour de très nombreuses pathologies jusqu'alors considérées comme hors de portée de la thérapeutique, qu'il s'agisse de maladies génétiques, de maladies dégénératives ou de diverses tumeurs. Largement favorisées par le développement des techniques de biologie et de génétique moléculaires, la mise au point et l'étude de modèles animaux de maladies, génotypiques et phénotypiques, ont été très fortement stimulées, de même que, dans la recherche clinique, l'élaboration de techniques d'évaluation.

Malgré ces acquis incontestables, le doute commence à s'installer quant aux réelles perspectives thérapeutiques que pourrait offrir le transfert de gène, dans un délai suffisamment proche en tout cas pour justifier des investissements massifs. Même s'il ne s'agit que d'un paramètre bien indirect pour l'évaluer, il est intéressant de remarquer que le facteur d'impact des deux grands journaux de thérapie génique (Gene Therapy et Human Gene Therapy) qui tournait depuis leur création autour de 8 et était encore à 7,4 en 1996 n'était plus en 1997, respectivement, que de 6 et de 6,3. Quelle que soit la valeur exacte de ce marqueur, la pente apparaît descendante. Après avoir largement recruté chercheurs et laboratoires des années durant, le champ de la recherche en thérapie génique est-il en train de perdre de son attrait ? Le « rapport Varmus » était déjà destiné à répondre à une certaine désillusion. Comme le notait Axel Kahn en janvier 1996, dans l'éditorial d'un numéro de médecine/sciences consacré à la thérapie génique: "Ainsi apparâ̂t-il que des efforts substantiels restent à accomplir avant de transformer la thérapie génique en une thérapie crédible pour le futur » [7]. Il était facile, toutefois, de dire que le début de l'expérience était alors trop proche pour que l'on puisse s'inquiéter. Il y avait à l'époque 150 essais cliniques enrôlant en tout un peu plus de 1000 personnes. On en compte trois ans plus tard, suivant les derniers recensements de la banque de données collectée par Wiley (http:// www.wiley.co.uk/genetherapy/clinical), près de 370 impliquant 3200 patients. Pour le moins, le moratoire sur les essais cliniques auquel appelait le "rapport Varmus » n'a eu que des effets modestes, et il est intéressant de noter que les trois quarts de ces essais ont lieu aux États-Unis, grâce aux fonds que distribue le NIH !

Ces essais cliniques sont, sauf rares exceptions, des études pilotes de "phase I/II", comme on le dit aujourd'hui, en utilisant un terme peu réglementaire pour indiquer qu'il s'agit en même temps d'analyser la tolérance et la faisabilité du geste, et de rechercher une indication d'efficacité, tout cela chez un très petit nombre de patients suivis en « ouvert» sans groupe témoin. Malgré ce caractère affiché d'étude pilote, on ne peut qu'être frappé par le décalage entre le nombre élevé d'essais et de patients et le manque d'ampleur des résultats publiés, du moins en termes de bénéfice clinique apporté au patient. En effet, mise à 
part la spectaculaire amélioration enregistrée chez des patients présentant une artérite des membres inférieurs compliquée, à la suite de l'administration intramusculaire d'un vecteur d'expression plasmidique contenant le gène du facteur angiogénique VEGF (vascular endothelial growth factor) [8], les bilans cliniques sont, pour le moins, peu enthousiasmants. Même, et il s'agit de cas exceptionnels, lorsque des bénéfices sont obtenus, comme chez certains patients atteints d'un glioblastome dans l'étude réalisée par l'équipe de David Klatzmann [9], ceux-ci semblent difficiles à reproduire dans des études pourtant apparemment semblables (la presse vient ainsi d'annoncer l'arrêt, faute de résultats significatifs, d'une large étude de phase III utilisant la même technique dans la même indication, organisée par Novartis). On peut s'interroger sur les causes de l'échec massif des essais de thérapie génique, et sur les difficultés rencontrées à reproduire les résultats positifs éventuellement obtenus chez de rares patients. Sans trop de crainte de se tromper, il est possible d'imputer un bon nombre de ces échecs à des erreurs d'élaboration des essais, ou à des défauts techniques qu'il est, ou devrait prochainement être, possible de corriger. On ne peut toutefois s'empêcher d'envisager, aussi, de véritables barrières biologiques et physiologiques, que nos techniques de transfert de gène ne sont pas aujourd'hui capables de franchir. Ce sont ces barrières, si elles existent, qu'il nous faut absolument identifier et tenter de franchir grâce à l'approfondissement de nos connaissances et de nos techniques. Il est sûrement difficile pour des chercheurs d'accepter de reconnaître une erreur (voire plusieurs) dans l'élaboration d'un essai clinique. Pourtant, ce serait sans doute là, au stade où nous en sommes, le meilleur apport que chacun devrait s'attacher à faire pour que le domaine avance et que la technique, si elle en a la capacité, émerge enfin. Alors que nous sommes potentiellement à la tête d'une base de données concernant plusieurs centaines d'essais et plusieurs milliers de patients, la tâche essentielle aujourd'hui est d'identifier les causes techniques d'échec, d'en définir les retombées, pour mieux les écarter et découvrir les obstacles que les cellules et l'organisme posent réellement devant l'approche thérapeutique.

Le « rapport Varmus » et l'éditorial d'Axel Kahn, il y a trois ans, avaient une conclusion commune, appelant à concentrer les efforts sur la recherche expérimentale, en particulier sur les questions de vectorisation et de voies d'apport. Une autre conclusion - complémentaire plutôt qu'alternative - s'impose trois ans après : pour que la thérapie génique ait un avenir clinique, il nous faut comprendre pourquoi son passé et son présent cliniques sont si décevants, que les chercheurs et les cliniciens impliqués dans les essais thérapeutiques trouvent, pour chacun de leurs échecs, les raisons pour lesquelles, ça ne marche pas. C'est largement à ce travail que se sont attelés les auteurs des synthèses publiées dans ce numéro de médecine/sciences et l'on peut espérer que cet exemple sera suivi.

La thérapie génique a été vécue comme un rêve, par les chercheurs, par les médecins, par les patients, et aussi par les industriels et les financiers. Nous voici revenus à la dure réalité scientifique

\section{RÉFÉRENCES}

1. Rosenberg SA, Aebersold P, Cornetta K, Kasid A, Morgan RA, Moen R, et al. Gene transfer into humans : immunotherapy of patients with advanced melanoma using tumor-infiltrating lymphocytes modified by retroviral gene transduction. $N$ Engl J Med $1990 ; 323: 570-8$.

2. Blaese RM, Culver KW, Miller AD, Carter CS, Fleischer T, Clerici M, et al. T lymphocyte-directed gene therapy for ADA-SCID : initial trial results after 4 years. Science 1995 ; $270: 475-80$.

3. Ross G, Erickson R, Knorr D, et al. Gene therapy in the United States : a five-year status report. Hum Gene Ther 1996 ; 7 : 1781-90.

4. Walsh C, Cepko CL. Clonally related cortical cells show several migration patterns. Science $1988 ; 241$ : 1342-5.

5. Maletic-Savatic M, Malinow R, Svoboda K. Rapid dendritic morphogenesis in CA1 hippocampal dendrites induced by synaptic activity. Science 1999 ; 283 : 1923-7.

6. Lisovoski F, Akli S, Peltekian E, et al. Phenotypic alteration of astrocytes induced by ciliary neurotrophic factor in the intact adult brain, as revealed by adenovirus-mediated gene transfer. J Neurosci 1997 ; 17 : 7228-36.

7. Kahn A. Thérapie génique : le temps d'un premier bilan. Med Sci $1996 ; 12$ : 9-12.

8. Baumgartner I, Pieczek A, Manor O, Blair R, Kearney M, Walsh K, Isner JM. Constitutive expression of phVEGF165 after intramuscular gene transfer promotes collateral vessel development in patients with critical limb ischemia. Circulation 1998; 97 : 1114-23.

9. Klatzmann D, Valery CA, Bensimon G, et al. A phase I/II study of herpes simplex virus type 1 thymidine kinase «suicide " gene therapy for recurrent glioblastoma. Hum Gene Ther 1998 ; 9 : 2595-604.

\section{TIRÉS À PART}

M. Peschanski.

\title{
Méthodes de base en génétique de la levure et biologie moléculaire
}

\author{
Informations: Pr Serge Potier et Pr Jean-Luc Souciet, UPRES-A 7010, \\ Université Louis-Pasteur/Cnrs \\ Institut de Botanique, 28, rue Goethe, 67000 Strasbourg, France \\ Fax : +33 3883584 84, @mail : souciet@gem.u-strasbg.fr
}

